\title{
Un Programa de Intervención Individualizado con Entrenadores de Fútbol
}

\section{An Individualized Intervention Program with Grass-root Soccer Coaches}

\section{Um Programa para Intervençáo Individualizada para Treinadores de Futebol}

\author{
Gerard Soriano, Yago Ramis, Jaume Cruz, Catarina Sousa
}

Universitat Autònoma de Barcelona

\begin{abstract}
Resumen: El objetivo del presente estudio fue el diseño y evaluación de un programa de intervención individualizado para fomentar un estilo de comunicación más positivo en los entrenadores y favorecer la satisfacción de las necesidades psicológicas básicas de los jugadores. Este estudio incorpora la mini teoría de las necesidades psicológicas básicas de la teoría de la autodeterminación en los programas individualizados con entrenadores. Utilizamos el CBAS como medida observacional para la evaluación de la conducta de los entrenadores y una escala para evaluar la percepción de satisfacción de las necesidad de autonomía de los jugadores. En el estudio participaron tres entrenadores de fútbol de 27, 22 y 33 ańos de edad y 54 jugadores con edades comprendidas entre los 12 y los 14 años $(M=12,9$; $D T=, 54)$. Tras la intervención los tres entrenadores lograron el objetivo de reducir las conductas punitivas. En cuanto a los objetivos relacionados con la dimensión de apoyo y de satisfacción de la necesidad de autonomía dos de los tres entrenadores lograron su consecución. La intervención individualizada resultó efectiva para el logro de los objetivos propuestos en dos de los tres entrenadores.

Palabras clave: teoría de la autodeterminación, estilo de comunicación, observación, necesidades psicológicas básicas.

Abstract: The purpose of the present study was to design and evaluate an individualized intervention program to promote coaches' positive communication style to favor players' basic psychological needs' satisfaction. This study is framed on the basic psychological needs theory from the self determination theory framework. We used CBAS as an observational measure to evaluate coaches' behavior and one scale to evaluate player's perceived satisfaction of autonomy. Three football coaches of 27, 22 and 33 years old, and their players with an age range between $12-14$ years old $(M=12.9 ; S D=$
\end{abstract}

.54) participated in the study. The three coaches achieved their behavioral goal to reduce punishment behaviours once the intervention. However for the behavioral goal related with support dimensión and players autonomy satisfaction goals, two of three coaches achieved their objectives. Individualized intervention was effective in achieving the objectives in two of the three coaches.

Keywords: self determination theory, communication style, observation, basic psychological needs.

Resumo: O objetivo do presente estudo foi desenhar e avaliar um programa de intervenção individualizado para promover um estilo de comunicaçáo positivo dos treinadores, de forma a satisfazer as necessidades psicológicas básicas dos jogadores. Este estudo integra o mini teoria das necessidades psicológicas básicas da teoria da autodeterminação em programas individualizados com treinadores. Foi utilizado o CBAS como medida observacional para avaliar o comportamento dos treinadores e uma escala para avaliar a percepção de satisfação da necessidade de autonomia dos jogadores. Participaram neste estudo três treinadores de futebol 27, 22 e 33 anos e os seus jogadores com idades compreendidas entre os 12 e os 14 anos $(M=$ $12,9 ; S D=0,54)$. Após a intervenção, os três treinadores alcançaram os objetivos que tinham estabelecido em quanto à mudança de comportamento punitivo. Quanto aos objectivos relacionados com a dimensão de apoio e de satisfação de autonomia dois dos três treinadores ganharam a sua realização. Intervenção individualizada foi eficaz na consecução dos objectivos em dois dos três treinadores.

Palavras-chave: teoria da autodeterminação, estilo de comunicação, observação, necessidades psicológicas básicas, treinadores.

\section{Introducción}

Los programas de formación para entrenadores se han caracterizado mayoritariamente por sesiones grupales en formato seminario. No obstante, los programas personalizados han demostrado las ventajas de adecuar los contenidos de enseñanza a las necesidades de cada entrenador (Cruz, Torregrosa, Sousa, Mora, y Viladrich, 2011) y sus efectos son más estables a largo plazo (Gilbert, Gallimore y Trudel 2009). Un ejemplo de estos programas es el Programa de Asesoramiento Personalizado a Entrenadores (PAPE; Sousa, Smith y Cruz, 2008; Sousa, Cruz, Torregrosa, Vilches y Viladrich, 2006). El PAPE

Dirección para correspondencia [Correspodence address]: Gerard Soriano. Facultat de Psicologia, Departament de Psicologia Bàsica, Evolutiva i de l' Educació. Universitat Autònoma de Barcelona, 08913 Bellaterra, Barcelona (Espańa). E-mail: gerard.soriano@uab.cat constituye una adaptación e individualización de las directrices conductuales del Coach Effectiveness Training (CET; Smith, Smoll y Curtis, 1979) por ello se intervenía de forma individual con el objetivo de incidir sobre las necesidades específicas de cada entrenador preservando su propio estilo. Inicialmente se entrevistaba a los entrenadores para conocer sus creencias y características personales y estimar en qué medida los conceptos que planteaba la intervención eran acordes con la forma de pensar de cada entrenador. Seguidamente se observaba la conducta del entrenador en los partidos mediante el Coaching Behavior Assesment System (CBAS, Smith, Smoll y Hunt, 1977), de este modo se generaba un perfil conductual en el que basar las sesiones de formación individualizadas. Los entrenadores hacían uso del establecimiento de objetivos 
para elegir las conductas a mejorar y planificar su consecución. El PAPE ha demostrado su efectividad en cuanto a la mejora de las conductas que los entrenadores establecieron como objetivo prioritario. Específicamente un aumento en aquellos comportamientos que suponían la implementación de conductas de apoyo, como el refuerzo o el ánimo, y una disminución de las conductas punitivas.

Estudios de revisión recientes (Cruz, et al., 2011; Langan, Blake, y Lonsdale, 2013) han apoyado la línea de intervención del PAPE, destacando la importancia de las intervenciones individualizadas, aunque también han señalado aspectos a tener en cuenta como la necesidad de aumentar el número de observaciones en la línea base y después de la intervención y la incorporación de nuevos enfoques teóricos como la Teoría de la Autodeterminación (Self Determination Theory - SDT, Deci y Ryan, 1985) por su relevancia en el campo de la psicología del deporte.

La Teoría de la Autodeterminación, desarrollada por Deci y Ryan $(1985 ; 2000)$ postula que los seres humanos somos organismos activos en busca del desarrollo y crecimiento personal. Para la SDT la motivación intrínseca i la internalización son procesos naturales que requieren de unos nutrientes para funcionar de manera óptima. Este el papel activo de las personas en la construcción de su crecimiento personal, integridad y bienestar se explica a partir de la mini teoría de las necesidades psicológicas básicas (Basic Psychological Needs - BPN por sus siglas en inglés). Las BPN son los nutrientes y el entorno y las personas que lo conforman son un elemento fundamental en la facilitación, o por el contrario en la frustración de estas necesidades. La satisfacción de las BPN (i.e., autonomía, competencia y relación) es esencial para el desarrollo psicosocial y el bienestar psicológico (Deci y Ryan, 2000) promoviendo mayor vitalidad subjetiva, autoestima y satisfacción con la vida (Balaguer, Castillo y Duda, 2008; Balaguer et al., 2012), mientras que la no satisfacción y la frustración de las BPN se ha relacionado con el malestar psicológico y mayores niveles de burnout (Castillo, González, Fabra, Mercé, y Balaguer, 2012).

Las intervenciones basadas en la SDT se han llevado a cabo en diversos ámbitos como el educativo (e.g., Reeve, 2002; Vansteenkiste, Simons, Lens, Soenens, y Martos, 2005), el del ejercicio físico (e.g., Markland, 1999; Wilson y Rodgers, 2004) pero todavía falta evidencia acerca de intervenciones llevadas a cabo en deporte. Los programas de formación que han incorporado aspectos de la SDT han sido implementados mayoritariamente en el ámbito de la educación física. Su y Reeve (2011) llevaron a cabo un estudio de meta-análisis analizando 19 programas de intervención basados en la SDT para explicar por qué algunas intervenciones eran más efectivas que otras. Estos autores señalaron que la limitación en la mayoría de programas era focalizar más en la teoría que en la práctica y el desconocimiento de las creencias, valores y personalidad de los participantes que podían entrar en conflicto con el mensaje dado. Este hecho apunta la necesidad de individualizar las intervenciones siguiendo la línea del PAPE.

Considerando los beneficios asociados a los programas in dividualizados con entrenadores (Cruz et al., 2011; Langan et al., 2013; Su y Reeve, 2011), la importancia de una base teórica sólida en la que fundamentar sus contenidos (Cruz et al., 2011; Langan et al., 2013) y la escasez de trabajos de intervención con SDT en el ámbito deportivo, el objetivo del estudio fue el diseño de un programa de formación a entrenadores basado en el PAPE y sustentado en la teoría de las BPN de la SDT, con el propósito de modificar la conducta de los entrenadores y satisfacer las necesidades psicológicas básicas de los jugadores partiendo de los objetivos planteados por cada entrenador.

\section{Método}

\section{Participantes}

Se seleccionaron tres entrenadores de fútbol con más de cinco años de experiencia. El entrenador 1 (E1) tenía 22 años de edad, seis de experiencia como entrenador, no disponía de titulación y estudiaba ciencias de la actividad física y el deporte. El entrenador 2 (E2) tenia 27 años de edad, diez de experiencia como entrenador, poseía el título de $1 \mathrm{r}$ nivel de entrenador de la Federación Catalana de Futbol (FCF) y era licenciado en Económicas. El entrenador 3 (E3) tenia 33 años de edad, 13 de experiencia como entrenador, poseía el título de monitor de fútbol base de la FCF y había estudiado un ciclo formativo de electricidad.. En el estudio también participaron 54 jugadores infantiles $(M$ edad $=12,9 ; D T=$ ,54) que respondieron el cuestionario.

\section{Instrumentos}

Medidas

Observación. Como medida observacional para registrar los comportamientos de los entrenadores se utilizó el Coaching Behavior Assesment System (CBAS, Smith, Smoll y Hunt, 1977) en su adaptación al español (Sousa et al., 2006) basada en tres dimensiones conductuales para evaluar la reacción del entrenador ante los aciertos y los errores de los jugadores: dimensión de Apoyo que incluye las categorías del CBAS Refuerzo (i.e., reacción positiva verbal o no verbal ante una buena jugada) y Ánimo al Error (i.e., el entrenador anima después de un error cometido), dimensión Punitiva que incluye las categorías de Castigo (i.e., reacción negativa verbal o no verbal después de un error) e Instrucción Técnica Punitiva (i.e., instrucción técnica después de un error dada de manera hostil o en forma de castigo) y la dimensión de que incluye las 
categorías de Instrucción Técnica al Error (i.e., Instrucción o demostración de cómo corregir el error cometido por el deportista) e Instrucción Técnica General (i.e., instrucción transmitida de forma espontánea respecto a la técnica o estrategia de juego).

Cuestionario. La necesidad de autonomía se evaluó con cinco ítems de la adaptación espańola (Alcaraz, Viladrich, y Torregrosa, 2013) del cuestionario Learning Climate Questionnaire de Standage, Ntoumanis y Duda (2005). Esta escala se ha utilizado en otros estudios para evaluar la necesidad de autonomía (e.g., Alcaraz et al, 2013). Los participantes respondieron a ítems como: "He decidido los ejercicios que he practicado". Los ítems fueron introducidos por la frase: "Durante las últimas 3 o 4 semanas, en este equipo de futbol...”. Las respuestas recogían el grado de acuerdo con los ítems planteados en una escala Likert-5, (1= Muy en desacuerdo; $5=$ Muy de acuerdo). Altas puntuaciones (i.e., 4 y 5) indicaban percepción de satisfacción de la necesidad de autonomía de los jugadores.

\section{Procedimiento}

La obtención de datos se llevó a cabo de acuerdo a las recomendaciones éticas de la American Psychological Association. Este estudio se planteó en tres fases: (1) línea base, (2) intervención y (3) evaluación de la intervención. Previo al análisis observacional se hizo un primer contacto para conocer a los entrenadores, éstos fueron informados de los objetivos del estudio y aceptaron participar voluntariamente bajo consentimiento informado.

Línea Base. Los jugadores completaron el cuestionario de satisfacción de la necesidad de autonomía y los entrenadores fueron grabados en tres partidos, por lo que obtuvimos nueve grabaciones en total. La grabaciones se realizaron durante la primera vuelta de la competición entre los meses de noviembre y diciembre. Como medida de control, para cada equipo, se grabaron dos partidos en condición de visitante y uno de local. Posteriormente cinco observadores con experiencia en la observación de entrenadores mediante el CBAS analizaron los datos. Tanto los datos observacionales de los entrenadores como los obtenidos de los cuestionarios administrados a los jugadores, sirvieron para orientar la intervención individualizada de acuerdo a las características de cada caso.

Intervención. El objetivo en la fase de intervención fue formar a los entrenadores en la creación de climas motivacionales adaptativos y en estilo de comunicación positivo. Para ello se realizaron tres sesiones, dos de ellas grupales y una individualizada. La sesiones fueron creadas por nuestro equipo de investigación, el Grup d'Estudis de Psicología de l'Esport (GEPE) de la Universidad Autónoma de Barcelona (UAB) con amplia experiencia en el diseño de intervenciones (e.g., PAPE). Las sesiones se realizaron en el mes de febrero dejando transcurrir una semana entre ellas y fueron impartidas por el primer autor, miembro del GEPE y entrenador de fútbol. En la primera sesión titulada Mejorando el clima motivacional se trató la importancia de un clima de apoyo a la satisfacción de las necesidades psicológicas básicas y se compartieron estrategias para ponerlo en práctica. En la segunda sesión titulada Directrices para mejorar la comunicación nos basamos en Smoll y Smith (1989) para fomentar un estilo de comunicación positivo con el objetivo de que los entrenadores identificasen las reacciones positivas y negativas ante los aciertos o los errores de sus jugadores y sus consecuencias. La tercera sesión, Recomendaciones para mejorar tu estilo de comunicación y satisfacer las necesidades de tus jugadores fue individualizada, siguiendo la línea del PAPE, el objetivo de esta sesión fue presentar a los entrenadores su perfil conductual y que se propusiesen sus propios objetivos de mejora. Los entrenadores se plantearon tres objetivos, de los cuales, dos eran conductuales y uno de satisfacción de necesidades. En la Tabla 1 se presentan los contenidos y duración de las sesiones.

Tabla 1. Estructuración del programa de intervención

\begin{tabular}{|c|c|c|c|c|}
\hline Sesión & Título & Contenido & Formato & Duración \\
\hline 1 & Mejorando el clima motivacional & $\begin{array}{l}\text { - Clima Motivacional } \\
\text { - SDT (Satisfacción de Necesidades) }\end{array}$ & Grupal & $1,5 \mathrm{~h}$ \\
\hline 2 & Directrices para mejorar la comunicación & - El estilo de comunicación positivo. & Grupal & $1,5 \mathrm{~h}$ \\
\hline 3 & $\begin{array}{l}\text { Recomendaciones para mejorar tu estilo } \\
\text { de comunicación y satisfacer las necesida- } \\
\text { des de tus jugadores }\end{array}$ & $\begin{array}{l}\text { - Recordatorio sesión } 1 \text { y } 2 . \\
\text { - Videofeedback y presentación del perfil. } \\
\text { - Establecimiento de objetivos }\end{array}$ & Individual & $1,5 \mathrm{~h}$ \\
\hline
\end{tabular}

Evaluación de la intervención. En la fase de evaluación de la intervención grabamos tres partidos de cada entrenador, en este caso se grabaron dos partidos jugando como local y uno como visitante. Los partidos fueron grabados durante la segunda vuelta del campeonato, entre los meses de abril y mayo, dejando pasar un mes después de la intervención. El objetivo de esta fase fue comparar los resultados obtenidos en la línea base con los de la post-intervención. 


\section{Estrategia de Análisis}

El registro de los datos observacionales se llevó a cabo mediante el software Lince (Gabín, Camerino, Anguera y Castañer, 2012) y se compararon las conductas emitidas por los entrenadores antes y después de la intervención. El cálculo del índice de acuerdo inter-observadores se obtuvo de la codificación del mismo partido realizada por los cinco observadores. Los resultados en la escala de satisfacción de autonomía de los jugadores se analizaron mediante el paquete estadístico SPSS v17.0. Para ello, se han agrupado los ítems de la escala y se han comparado los porcentajes de respuesta en las categorías 4 y 5 del cuestionario en la línea base y en la Post intervención.

\section{Resultados}

Los resultados conductuales de los entrenadores se presentan en dos fases (i.e., línea base y post-intervención). En la primera se presenta el perfil conductual de los entrenadores y los objetivos de cambio (i.e., dos objetivos conductuales y uno de satisfacción de necesidades) planteados por cada entrenador. En la segunda fase se presenta el cambio conductual de cada entrenador y finalmente el grado de logro de los dos objetivos conductuales planteados. Finalmente, respecto a las Necesidades Psicológicas Básicas se presentan los porcentajes de respuesta al cuestionario en la línea base y post intervención, y el grado de logro de los objetivos relacionados con las BPN que se habían propuesto los entrenadores. Para el cuestionario, el porcentaje de valores faltantes estuvo por debajo del $5 \%$, por lo que no afectaron al análisis de los datos (Graham, 2009). La fiabilidad de los datos observacionales mediante el índice de acuerdo inter-observadores calculado con el coeficiente Kappa de Cohen fue buena (0,66), según criterio de Landis y Koch (1977).

\section{Entrenador 1}

Línea base. Se codificaron 991 conductas de las cuales (249; 25,13\%) eran de Apoyo, Punitivas (199; 20,08\%) y de Instrucción $(543 ; 54,79 \%)$ lo que sugiere un perfil de entrenador con predominio de las instrucciones y con valores similares en cuanto a conductas de apoyo y punitivas (véase Figura 1). Los objetivos de cambio planteados por el E1 fueron los siguientes: (1) Reducir las conductas punitivas sustituyendo las instrucciones técnicas punitivas por instrucciones técnicas al error (2) Aumentar el apoyo (3) Dar más autonomía a los jugadores.

Post-intervención. Después de la intervención se codificaron 1248 conductas, lo que representa un aumento respecto a la línea base. Tal como se observa en la Figura 1 se registró un aumento en la frecuencia de conductas de apoyo (de 249 a $275) ;(25,13 \%$ a $22,04 \%)$ una ligera disminución de las con- ductas de castigo (de 199 a 187); (20,08\% a 14,98\%) y un gran aumento de instrucciones (de 543 a 786); (54,79\% a 62,98\%). En cuanto a los objetivos conductuales planteados el entrenador logró reducir el porcentaje de conductas punitivas pero no logró un aumento del porcentaje de conductas de apoyo. El aumento en las instrucciones se explica por la reducción de instrucciones técnicas punitivas (i.e., subcategoría de la dimensión Punitiva) a favor del aumento de instrucciones técnicas al error (subcategoría de la dimensión de Instrucción).

Figura 1. Resultados de las observaciones del entrenador 1 antes y después de la intervención expresados en porcentaje y frecuencia absoluta.

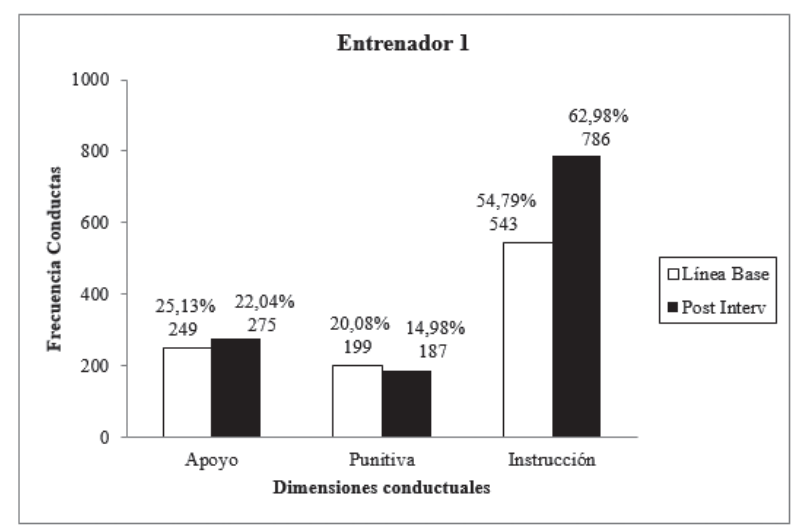

\section{Necesidades psicológicas Básicas}

Tal como se observa en la Figura 2 el porcentaje de jugadores que puntuaron valores altos en la escala de satisfacción de la necesidad de autonomía disminuyó respecto a la línea base (de 64,4\% a 48,4\%). Por lo que los jugadores tuvieron menor percepción de autonomía y el E1 no logró el objetivo 3.

Figura 2. Porcentaje de jugadores que perciben satisfacción de autonomía antes y después de la intervención

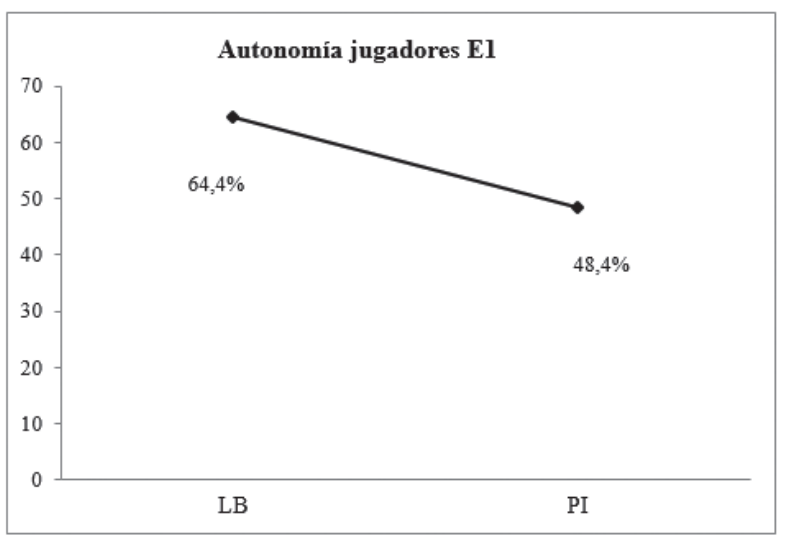




\section{Entrenador 2}

Línea base. Se codificaron 1285 conductas. En la dimensión de Apoyo (385; 29,96\%), en la dimensión Punitiva (302; $23,50 \%)$ y en la de Instrucción $(598 ; 46,54 \%)$. Estos resultados sugieren un perfil de entrenador donde predominan las instrucciones y con un elevado número de conductas punitivas, aunque las conductas de apoyo fueron superiores a las punitivas (véase Figura 2). Los objetivos de cambio conductual planteados por el E2 fueron los siguientes: (1) disminuir el número de conductas de castigo, (2) aumentar las conductas de apoyo reforzando ante los aciertos y animando ante los errores y (3) Favorecer la autonomía.

Post-intervención. Tras la intervención el E2 mostró menor número de conductas (de 1285 a 813). Tal como se observa en la Figura 3 el entrenador redujo el número de conductas de apoyo respecto a la línea base (de 385 a 245) aunque el porcentaje fue ligeramente superior $(29,96 \%$ a $30,14 \%)$, logrando de este modo la consecución del objetivo 2 . Las conductas de castigo disminuyeron drásticamente (de 302 a 49); $(23,50 \%$ a $6,03 \%)$, lo que coincide con el logro del objetivo 1 . Las conductas de instrucción también disminuyeron de frecuencia (de 598 a 519) aunque el porcentaje fue muy superior (de $46,54 \%$ a $63,84 \%$ ).

Figura 3. Resultados de las observaciones del entrenador 2 antes y después de la intervención expresados en porcentaje y frecuencia absoluta.

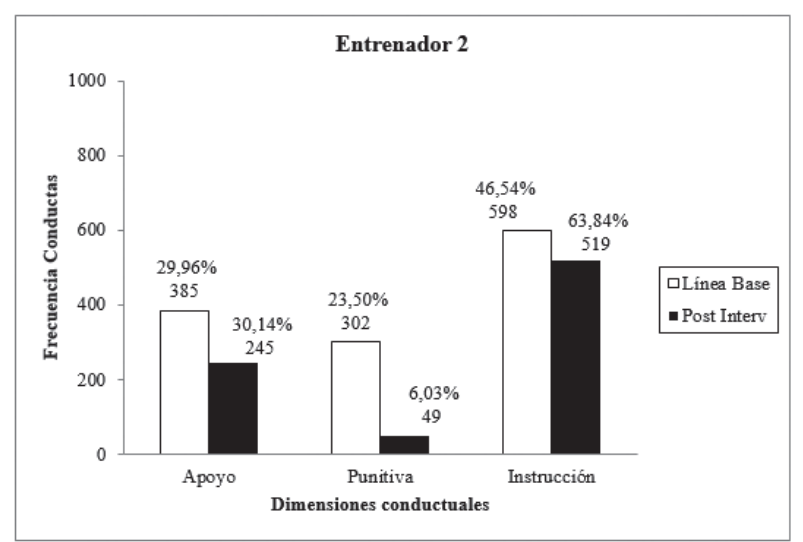

\section{Necesidades psicológicas Básicas}

El porcentaje de jugadores que perciben satisfacción de autonomía aumenta después de la intervención, por lo que el E2 logra el objetivo de favorecer la autonomía.
Figura 4. Porcentaje de jugadores que perciben satisfacción de autonomía antes y después de la intervención LB- Línea Base; Post- Fase post intervención.

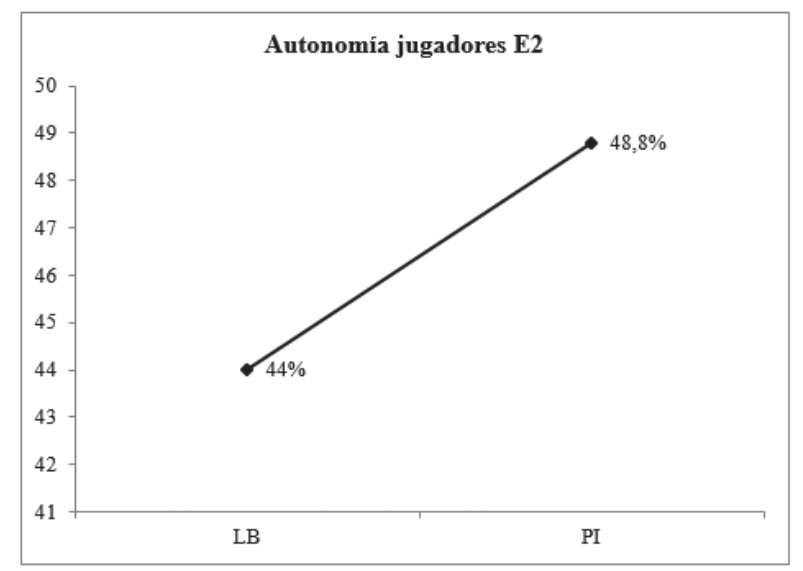

Entrenador 3

Línea base. Se codificaron 1099 conductas. En la dimensión de Apoyo (216; 19,65\%), Punitivas (257; 23,38\%) y de Instrucción $(626 ; 56,96 \%)$, lo que sugiere un perfil de entrenador punitivo registrando más conductas punitivas que de apoyo (véase Figura 3). Los objetivos de cambio conductual planteados por el E3 fueron los siguientes: (1) dejar de ser un entrenador punitivo reduciendo el número de conductas en esta dimensión, (2) Aumentar las conductas de apoyo reforzando ante los aciertos y animando ante los errores y (3) Fomentar la autonomía.

Post-intervención. Tras la intervención el E3 aumentó el número de conductas (de 1099 a 1547). Tal como se observa en la Figura 5 el entrenador aumentó el número de conductas de apoyo respecto a la línea base (de 216 a 457); (19,65\% a $29,54 \%$ ), logrando la consecución del objetivo 2 . Las conductas de castigo disminuyeron (de 257 a 145); (23,38\% a $9,37 \%$ ), lo que coincide con el logro del objetivo 1 . Las conductas de instrucción aumentaron (de 626 a 945); (56,96\% a $61,08 \%)$. 
Figura 5. Resultados de las observaciones del entrenador 3 antes y después de la intervención expresados en porcentaje y frecuencia absoluta.

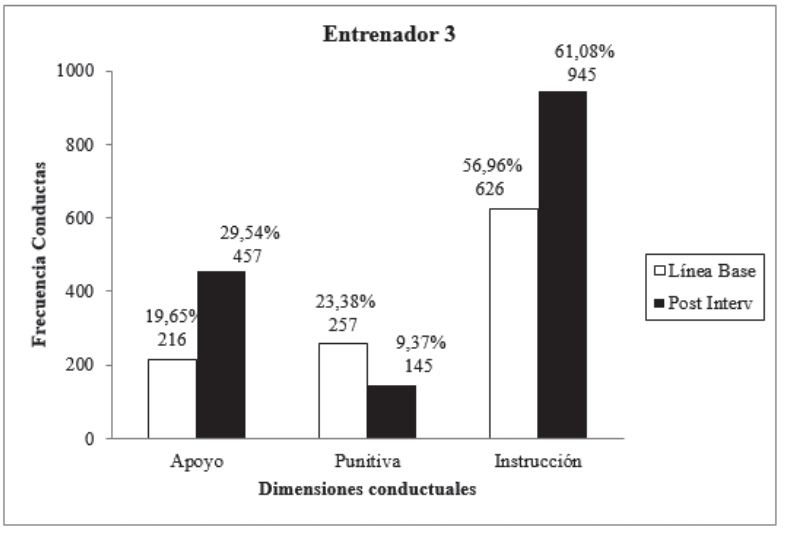

Necesidades Psicológicas Básicas

Tal como se observa en la Figura 6, después de la intervención un mayor número de jugadores percibieron satisfacción de la necesidad de autonomía (de $38,6 \%$ a $58,9 \%$ ) por lo que se cumplió el objetivo que se había propuesto su entrenador.

Figura 6. Porcentaje de jugadores que perciben satisfacción de autonomía antes y después de la intervención.

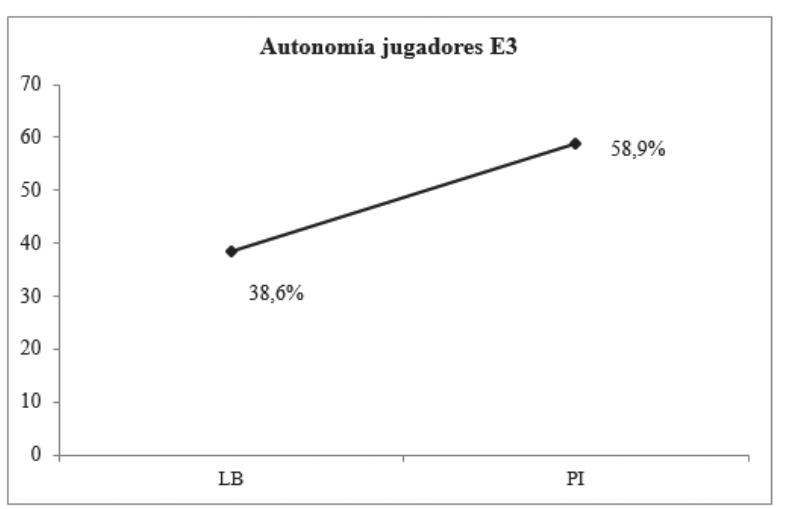

\section{Discusión}

En base a los objetivos planteados por cada entrenador, el propósito de este estudio fue el diseño y evaluación de un programa de intervención individualizado. Los resultados obtenidos ponen de manifiesto un logro general de los objetivos planteados por cada entrenador. En cuanto al perfil de los entrenadores, los datos observacionales de la línea base han revelado dos perfiles de entrenador con predominio de las conductas de instrucción y frecuencias similares en cuanto a conductas de apoyo y conductas punitivas (i.e., E1, E2) y un perfil de entrenador punitivo con mayor frecuencia de conductas punitivas que de apoyo (i.e., E3). Tras la intervención el perfil conductual de los entrenadores cambió. En el caso del E3 las conductas de apoyo superaron a las de castigo, para el E1 y el E2 aumentó la diferencia entre conductas punitivas y de apoyo, y en el caso del E2 además se redujo el número de instrucciones. En cuanto a los objetivos conductuales planteados los tres entrenadores logran reducir el número de conductas punitivas y dos de ellos consiguen aumentar el número de conductas de apoyo (i.e., E2 y E3).

En cuanto al objetivo de necesidades psicológicas, los tres entrenadores se plantearon el objetivo de apoyar la satisfacción de autonomía. Esto pudo deberse a lo novedoso que resultó el concepto de autonomía en los entrenadores, acostumbrados a adoptar metodologías de entrenamiento directivas que tienen en poca consideración la opinión de los jugadores. El aumento del porcentaje en la satisfacción de la necesidad de autonomía en la fase post sugiere el logro de los objetivos de satisfacción de la necesidad de autonomía de los jugadores que se habían propuesto los entrenadores, a excepción del equipo del E1, donde el porcentaje disminuye. Por lo que este entrenador no logra la consecución del objetivo.

De acuerdo con la revisión de Langan et al. (2013) que apoya la utilidad de programas individualizados, los principales resultados de este estudio apoyan la efectividad de la intervención respecto al logro de objetivos de los entrenadores. En línea con otros trabajos (e.g., Cruz et al., 2011; Sousa et al., 2008; Sousa et al., 2006) los tres entrenadores participantes lograron disminuir la frecuencia de conductas punitivas, siendo el E2 el que consiguió mayor mejora. En cuanto a las conductas de apoyo, los tres entrenadores se propusieron el objetivo de aumentarlas. Los entrenadores lograron este objetivo con excepción del E2, que disminuyó las conductas de apoyo, un hecho que contrasta con la reducción de conductas punitivas de este entrenador. Igual que en otros estudios (e.g., Boixadós y Cruz, 1999; Sousa et al., 2006) las conductas de instrucción fueron las más observadas.

En cuanto a las necesidades psicológicas en el ámbito educativo, la percepción de satisfacción de las BPN se ha asociado con consecuencias positivas como mayor participación en la clase, mayor desarrollo de habilidades o mejores calificaciones académicas (Cheon et al., 2012; Su y Reeve, 2011). En el ámbito del deporte de iniciación, Balaguer y colaboradores (2012) han asociado la satisfacción de las BPN con el bienestar de los deportistas (e.g., mayor vitalidad, menor burnout y menor tasa de abandono). Estos trabajos ponen de manifiesto la importancia de un entorno de apoyo a las BPN.

Igual que en el trabajo de Sousa et al. (2006) atendiendo a las características de nuestro trabajo creemos relevante la presentación de información cualitativa respecto a los entrenadores. Así el E1debido a aspectos como su nivel de formación (i.e., no posesión del título de entrenador), las caracte- 
rísticas del club al que entrenaba y los malos resultados en la segunda vuelta del campeonato en comparación a los de la primera vuelta, pudieron propiciar la no consecución del objetivo de aumentar el apoyo y favorecer la autonomía de los jugadores, aunque si logró el objetivo de reducir las conductas punitivas tomando conciencia de su estilo negativo a la hora de corregir errores y convirtiendo las instrucciones transmitidas de forma hostil en instrucciones sin carga negativa. El E2, era el de mayor formación tanto a nivel futbolístico como a nivel académico y se implicó a fondo en aplicar las estrategias que se habían trabajado en las sesiones de intervención, prueba de ello fue su interés por la psicología del deporte aprovechando las ocasiones en la que grabábamos los partidos para comentar aspectos a mejorar. Debido a su nivel formativo el nivel de exigencia a sus jugadores era elevado y acostumbraba a corregir de manera punitiva por lo que quiso emplearse en reducir estas conductas. Su equipo logró el ascenso de categoría de forma destacada. Estos aspectos pudieron incidir en la implicación del entrenador y en la consecución de los tres objetivos planteados. El E3 era el de más edad y mayor experiencia como entrenador, los resultados del equipo fueron negativos tanto en la primera vuelta como en la segunda. Antes de la intervención el entrenador se quejaba del nivel de sus jugadores, apoyaba poco y acostumbraba a dirigir sus instrucciones con más frecuencia a tres o cuatro jugadores del equipo. Finalmente el entrenador distribuyó las instrucciones de manera equilibrada hacia todos los jugadores y se centró en adoptar un enfoque positivo basado en el apoyo sobre las cosas que hacían bien sus jugadores más que en las conductas punitivas ante los errores. Estos aspectos influyeron en que el E3 lograse los tres objetivos que se había planteado.

Cabe mencionar ciertas limitaciones presentes en este estudio como la evaluación de la satisfacción dela necesidad de autonomía mediante cuestionarios que dificulta la obtención de puntuaciones más objetivas. Estudios posteriores han de incorporar otras medidas (e.g., observacional) tal como se está planteando recientemente en el ámbito educativo (Haerens et al., 2013). Nuestro estudio es de naturaleza descriptiva y con un número reducido de participantes, ciertamente los estudios de caso dificultan la inferencia de relaciones causales. Por ello siguiendo las recomendaciones de Cruz et al. (2011) se ha contrarrestado la ausencia de grupo control aumentando el número de observaciones en la línea base y en la fase post-intervención respecto otros estudios como el de Sousa et al. $(2008,2006)$.

\section{Aplicaciones prácticas}

Este trabajo supone una evidencia práctica dirigida a mejorar la calidad del entorno deportivo. Las intervenciones con entrenadores de deportistas jóvenes suponen un marco de trabajo excelente para mejorar la calidad del deporte de iniciación, mejorando la experiencia de los deportistas, favoreciendo el compromiso con la práctica deportiva y evitando su abandono a largo plazo.

Agradecimientos.- Este trabajo se ha realizado, en parte, gracias al proyecto I+D del Ministerio de Economía y Competitividad (DEP2010-15561) (subprograma DEPO).

\section{Referencias}

1. Alcaraz, S., Viladrich, C. y Torregrosa, M. (2013). Less time, better quality. Shortening questionnaires to assess team environment and goal orientation. Spanish Journal of Psychology, 16. doi: 10.1017/sjp.2013.74

2. Balaguer, I., Castillo, I. y Duda, J. L. (2008). Apoyo a la autonomía, satisfacción de las necesidades, motivación y bienestar en deportistas de competición: un análisis de la teoria de la autodeterminación. Revista de Psicología del Deporte, 17, 123-139.

3. Balaguer, I., González L., Fabra, P., Castillo, I., Mercé, J. y Duda, J. L. (2012). Coaches' interpersonal style, basic psychological needs and the well- and ill-being of young soccer players: A longitudinal analysis. Journal of Sport Sciences, 30 (15), 1619-1629

4. Boixadós, M. y Cruz, J. (1999). Intervención conductual en entrenadores de futbolistas alevines. En F. Guillén (Ed.), La Psicología del Deporte en España al final del milenio (pp. 423-431). Las Palmas de Gran Canaria: Servicio de Publicaciones de la Universidad de Las Palmas de Gran Canaria.

5. Castillo, I., González L., Fabra, P., Mercé, J. y Balaguer, I. (2012). Estilo interpersonal controlador del entrenador, frustración de las necesidades psicológicas básicas, y burnout en futbolistas infantiles. Cuadernos de Psicología del Deporte, 12 (1), 143-146.

6. Cheon, S., Reeve, J. y Moon I. (2012). Experimentally based, longitudinally designed, teacher-focused intervention to help physical education teachers be more autonomy supportive toward their students. Journal of Sport \& Exercise Psychology, 34, 365-396.
7. Cruz, J.; Torregrosa, M., Sousa, C., Mora, A. y Viladrich, C. (2011). Efectos conductuales de programas personalizados de asesoramiento a entrenadores en estilo de comunicación y clima motivacional. Revista de Psicología del Deporte. 20/1, 179-195.

8. Deci, E. L. y Ryan, R. M. (1985). Intrinsic motivation and self-determination in human behavior. New York: Plenum.

9. Deci, E. L. y Ryan, R. M. (2000). The "what" and "why" of goal pursuits: Human needs and the self-determination of behavior. Psychological Inquiry, 11, 227-268.

10. Gabín, B., Camerino, O., Anguera, Ma.T. y Castañer, M. (2012). Lince: multiplatform sport analysis software. Procedia Computer Science Technology, 46, 4692-4694.

11. Gilbert, W. D., Gallimore, R. y Trudel, P. (2009). A learning community approach to

12. coach development in youth sport. Journal of Coaching Education, 2(2), $1-21$.

13. Graham, J. W. (2009). Missing data analysis: making it work in the real world. Annual Review of Psychology, 60, 549-576. doi: 10.1146/annurev. psych.58.110405.085530

14. Haerens, L., Aelterman, N., Van den Berghe,L., De Meyer, L., Soenens, B. y Vansteenkiste, M. (2013). Observing Physical Education Teachers'Need-Supportive Interactions in Classroom Settings. Journal of Sport \& Exercise Psychology, 35, 3-17. 
15. Landis J.R. y Koch G.G. (1977) The measurement of observer agreement for categorical data. Biometrics, 33, 159-174.

16. Langan, E., Blake, C. y Lonsdale, C. (2013). Systematic review of interpersonal coach education interventions on athlete outcomes. Psychology of Sport and Exercise, 14, 37-49.

17. Markland, D. A. (1999). Self-determination moderates the effects of perceived

18. competence on intrinsic motivation in an exercise setting. Journal of Sport and

19. Exercise Psychology, 21, 351-361.

20. PASW Statistics (versión 17) [Programario informático]. Chicago, IL: SPSS Inc.

21. Reeve, J. (2002). Self-determination theory applied to educational settings. In

22. E. L. Deci, \& R. M. Ryan (Eds.), Handbook of self-determination research (pp.

23. 183-203). Rochester, NY: University of Rochester Press.

24. Smith, R. E., Smoll, F. L. y Hunt, E. (1977). A system for the behavioral assessment of athletic coaches. Research Quarterly, 48, 401-407.

25. Smith, R. E., Smoll, F. L. y Curtis, B. (1979). Coach efectiveness training. A cognitive behavioral approach to enhancing relationship skills in youth sports coaches. Journal of Sport Psychology, 1, 59-75.

26. Smith, R. E., Smoll, F. L. y Cumming, S. P. (2007). Effects of a motivational climate intervention for coaches on young athletes' sport performance anxiety. Journal of Sport and Exercise Psychology, 29, 39-59.
27. Smoll, F. y Smith, R. (1989). Leadership behaviors in sport: a theoretical model and research paradigm. Journal of Applied Social Psychology, 19 1552-1551.

28. Sousa, C., Cruz, J. Torregrosa, M., Vilches, D. y Viladrich, C. (2006). Evaluación conductual y programa de asesoramiento personalizado a entrenadores (PAPE) de deportistas. Revista de Psicología del Deporte, 15, 263-278.

29. Sousa, C., Smith, R. E. y Cruz, J. (2008). An individualized behaviora goal-setting program for coaches. Journal of Clinical Sport Psychology, 2, 258-277.

30. Su, Y-L. Y Reeve, J. (2011). A meta-analysis of the effectiveness of intervention programs designed to support autonomy. Educational Psychology Review, 23, 159-188. doi:10.1007/s10648-010-9142-7

31. Standage, M., Duda, J. L. y Ntoumanis, N. (2005). A test of self-determination theory in school PE. British Journal of Educational Psychology, 75, 411-433.

32. Vansteenkiste, M., Simons, J., Lens, W., Soenens, B. y Matos, L. (2005). Examining the impact of extrinsic versus intrinsic goal framing and in ternally controlling versus autonomy-supportive communication style upon early adolescents'

33. academic achievement. Child Development, 76, 483-501. doi:10.1111/ j.1467-8624.2005.00858.x

34. Wilson, P. M., y Rodgers, W. M. (2004). The relationship between perceived autonomy support, exercise regulations and behavioural intentions in women. Psychology of Sport and Exercise, 5, 229-242. 